\title{
Neural correlates of consciousness of sequence knowledge: A novel application of the process dissociation procedure
}

Article in Neurolmage · May 2000

DOI: 10.1016/S1053-8119(00)91336-3

\section{CITATIONS}

0

8 authors, including:

Arnaud Destrebecqz

Université Libre de Bruxelles

55 PUBLICATIONS 2,221 CITATIONS

SEE PROFILE

André Luxen

University of Liège

428 PUBLICATIONS 19,825 CITATIONS

SEE PROFILE
READS

64

\section{Pierre Maquet}

University of Liège

229 PUBlicATIONS 13,464 CITATIONS

SEE PROFILE

Martial Van der Linden

University of Geneva

771 PUBLICATIONS 22,766 CITATIONS

SEE PROFILE

Some of the authors of this publication are also working on these related projects:

Alcohol dependence View project

Schizophrenia: a cognitive approach View project 


\title{
Neural Correlates of Consciousness of Sequence knowledge: A Novel Application of the Process Dissociation Procedure
}

\author{
Arnaud Destrebecqz*, Philippe Peigneux $\nmid \neq$, Pierre Maquet $\dagger$, Christian Degueldre $\dagger$, \\ Guy Delfiore $\dagger$, André Luxen†, Martial Van der Linden*, Axel Cleeremans* \\ * Cognitive Science Research Unit, Université Libre de Bruxelles \\ †Cyclotron Research Centre, Université de Liège \\ ¥Neuropsychology Department, Université de Liège
}

\begin{abstract}
A central issue in the study of implicit learning and memory is to determine the extent to which newly acquired knowledge can be projected onto hehavior without being available to consciousness. To address this issue, this study offers a novel application of the process dissociation procedure (PDP; Jacoby, 1991) to sequence learning. Participants were first trained on a serial reaction time (SRT) task and subsequently asked to generate similar (inclusion) or different (exclusion) sequences of key presses. Continued generation of sequences of key presses similar to the training sequence under exclusion instructions would indicate that participants are not consciously aware of the relevant regularities. To identify the brain regions specifically involved in each condition, regional cerebral blood flow (rCBF) was recorded in a positon emission tomography (PET) experiment while participants performed the two generation tasks.
\end{abstract}

\section{Method}

Training Task. 17 normal right-handed subjects were trained on a four-choice SRT task during 15 blocks of 96 trials each prior to scanning. They were instructed to press with the right hand as fast and as accurately as possible on the key corresponding to the location of a target appearing on a screen (RSI $250 \mathrm{msec}$ ). Unknown to participants, each block contained 8 repetitions of a 12-element sequence entirely composed of second-order (SOC) conditional transitions. To assess the extent of sequence-specific learning, Block 13 contained a different $\mathrm{SOC}$ sequence.

Generation Task. After training, subjects were informed that the dots had followed a repeating pattern. During each of three 90 seconds PET scans, they were then asked to reproduce the training sequence or, failing recollection, to guess it based on their intuition (Inclusion [Incl] condition). Next, they were instructed to try to avoid reproducing the sequence in three subsequent scans (Exclusion [Excl] condition). Generation scores were computed for each condition as a percentage of all generated three-elements chunks (from the number of generated elements during the elapsed 1' $30^{\prime \prime}$ ) that respected the training sequence. Compared with a chance level of $33 \%$, subjects generated $71 \%$ of training triplets under inclusion and only $31 \%$ under exclusion $[\mathrm{F}(1,15)=41.254 ; p<.0001]$, indicating that their sequence knowledge was available to conscious awareness and control.

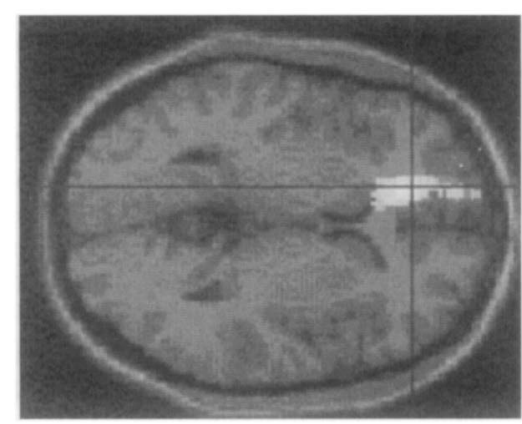

\section{Brain Mapping}

PET scans were obtained with a Siemens CTI $951 \mathrm{R} 16 / 31$ scanner in 3D mode.

The rCBF was measured using oxygen-15 labelled water infusions during Incl and Excl conditions. Using SPM99b, the specific rCBF response to the Condition [Incl vs Excl] by Performance (generation scores) interaction was estimated using a design matrix that included block effect and global activity as confounding covariates. At voxel-corrected level, significant correlated rCBF responses were located (see figure) in the depth of both anterior cingulate (BA 32) and medial frontal (BA 10) gyri (-14 $422 \mathrm{~mm}$, $\mathrm{Z}=4.83 ;-14380 \mathrm{~mm}, \mathrm{Z}=4.79$, ps corr. $<.05$ ).

\section{Conclusion}

The process dissociation procedure is a useful tool to ascertain the extent to which knowledge is available to consciousness. Our findings indicate that anterior cingulate and medial frontal cortex are specifically involved in supporting conscious control and recall of newly acquired sequence knowledge. Further studies will attempt to identify the brain regions specifically involved in supporting implicit knowledge. An open issue is whether these regions will involve neural networks distinct from those involved in this experiment.

\section{References}

1. Jacoby, L.L. A process dissociation framework: Separating automatic from intentional uses of memory J. Mem. Lang. 30 , 513-541.

2. SPM99b; Wellcome Department of Cognitive Neurology, Institute of Neurology, London, UK 